\title{
Automated Electronic Medical Record Sepsis Detection in the Emergency Department
}

Background: While often first treated in the Emergency Department (ED), identification of sepsis is difficult. Electronic medical record (EMR) clinical decision tools offer a novel strategy for identifying patients with sepsis. The objective of this study was to test the accuracy of an EMR-based, automated sepsis identification system. Methods: We tested an EMR-based sepsis identification tool at a major academic, urban ED with 64,000 annual visits. The EMR system collected vital sign and laboratory test information on all ED patients, triggering a "sepsis alert" for those with $\geq 2$ SIRS (systemic inflammatory response syndrome) criteria (fever, tachycardia, tachypnea, leukocytosis) plus $\geq 1$ major organ dysfunction (SBP $\leq 90 \mathrm{~mm} \mathrm{Hg}$, lactic acid $\geq 2.0 \mathrm{mg} / \mathrm{dL}$ ). We confirmed the presence of sepsis through manual review of physician, nursing, and laboratory records. We also reviewed a random selection of ED cases that did not trigger a sepsis alert. We evaluated the diagnostic accuracy of the sepsis identification tool. Results: From January 1 through March 31, 2012, there were 795 automated sepsis alerts. We randomly selected 300 cases without a sepsis alerts from the same period. The true prevalence of sepsis was 355/795 (44.7\%) among alerts and 0/300 (0\%) among non-alerts. The positive predictive value of the sepsis alert was 44.7\% (95\% Cl: 41.2-48.2\%). Pneumonia and respiratory infections (38\%) and urinary tract infection (32.7\%) were the most common infections among the 355 patients with true sepsis (true positives). Among false-positive sepsis alerts, the most common medical conditions were gastrointestinal (26.1\%), traumatic $(25.7 \%)$, and cardiovascular $(20.0 \%)$ conditions. Rates of hospital admission were: true-positive sepsis alert $91.0 \%$, false-positive alert $83.0 \%$, no sepsis alert 5.7\%. Conclusions: This ED EMR-based automated sepsis identification system was able to detect cases with sepsis. Automated EMR-based detection may provide a viable strategy for identifying sepsis in the ED. 
(Additional Revisions March 19, 2014)

$5 \quad$ Su Q. Nguyen, BS (1)

6 Edwin Mwakalindile, BS (1)

7 James S. Booth, MD (2)

8 Vicki Hogan, RN

9 Jordan Morgan, MPH (2)

10 Charles T. Prickett, MPH

11 John P. Donnelly, MSPH (2)

12 Henry E. Wang, MD, MS (2)

13 (1) University of Alabama School of Medicine

(2) Department of Emergency Medicine, University of Alabama School of Medicine, Birmingham, Birmingham, Alabama

Contact Information:

17 Henry E. Wang, MD, MS

18 Department of Emergency Medicine

19 University of Alabama at Birmingham

20619 19th Street South, OHB 251

21 Birmingham, AL 35249

22 (205) 996-6526

23 Fax (205) 975-4662

24 hwang@uabmc.edu

25 Word Count: 2,289 
27 Sepsis is the syndrome of microbial infection complicated by systematic inflammation. Sepsis

28 may subsequently lead to organ dysfunction, shock, and death.(Levy et al. 2003) Sepsis is a major public health problem, accounting for more than 750,000 hospital admissions, 500,000 emergency department (ED) visits and 200,000 deaths annually.(Angus et al. 2001; Annane et al.

31 2005; Jones 2006) Early aggressive therapy is essential for optimizing outcomes from sepsis.

32 (Rivers et al. 2001)

In recent years, physicians have increasingly utilized electronic medical records (EMR) systems to aid clinical decision making.(Levy \& Heyes 2012) By collecting and organizing clinical data, EMR systems have strong potential to improve the detection of conditions where symptoms or laboratory findings are difficult to discern. Diagnosis of sepsis is difficult because clinicians may not recognize the constellation of clinical, physiologic and laboratory abnormalities that comprise the syndrome. Several efforts have attempted to use EMR systems for sepsis detection, albeit with marginal results.(Jaimes et al. 2003; Nelson et al. 2011) A prominent limitation of these prior efforts was the absence of data for hypotension or lactic acidosis, which are often prominent features of sepsis and may indicate the need for aggressive protocolized resuscitation.(Rivers et al. 2001)

In this study we sought to evaluate the accuracy of an automated EMR sepsis detection system in the ED. 


\section{Study Design}

47 We conducted a retrospective analysis of automated clinical data collected by an ED EMR system. The study was approved via a written application by the Institutional Review Board of the University of Alabama at Birmingham (approval \#X120409014).

\section{Study Setting}

51 This study utilized ED data from the University of Alabama at Birmingham (UAB) Hospital, an

52 urban academic tertiary care referral medical center in Birmingham, Alabama, United States. The

53 ED treats over 64,000 patients annually and is the only Level I trauma center in Alabama. While

54 the ED does not restrict the age of treated patients, the ED population is predominantly $(>99 \%)$

55 adult. UAB Hospital has over 900 inpatient beds, including more than 180 critical care beds.

\section{EMR Sepsis Detection System}

The ED utilized the Cerner FirstNet ${ }^{\circledR}$ (Kansas City, Missouri) EMR system. The FirstNet system collects comprehensive demographic and clinical information for all patients presenting receiving care in the ED, including patient demographics, location and status in the ED, care time points,

60 laboratory and other test results, nursing and physician documentation, and patient education.

61 Access to the master database is facilitated using a proprietary database language (Cerner

62 Command Language) patterned after Structured Query Language (SQL). 
Using the Cerner FirstNet platform we developed an automated sepsis detection system. The sepsis detection system drew upon clinical and laboratory information documented on all ED patients. The detection system was developed using the Cerner Discern Analytics ${ }^{\circledR}$ v.2.0 reporting and data analysis tool, a Java-based program which is integrated with the EMR system.

The sepsis detection system triggered a "sepsis alert" if the EMR identified two or more Systemic Inflammatory Response Syndrome (SIRS) criteria and at least one sign of shock. SIRS criteria included 1) (temperature $\leq 36^{\circ} \mathrm{C}\left(96.8^{\circ} \mathrm{F}\right)$ or $\left.\geq 38^{\circ} \mathrm{C}\left(100.4^{\circ} \mathrm{F}\right), 2\right)$ respiratory rate $\geq 20$ breaths $/ \mathrm{min}$, 3) heart rate $\geq 90$ beats/min, and 4) total white blood cell (WBC) count $\leq 4,000$ or $\geq 12,000$ cells $/ \mathrm{mm}^{3}$, or $>10 \%$ bands. Signs of shock included 1) systolic blood pressure $\leq 90 \mathrm{~mm} \mathrm{Hg}$, or lactic acid $\geq 2.0 \mathrm{mg} / \mathrm{dL}$. We chose these definitions based upon criteria used by Rivers, et al. in a clinical trial of septic shock.(Rivers et al. 2001) While Rivers, et al. used a lactic acid lactate threshold $\geq 4.0 \mathrm{mg} / \mathrm{dL}$, we lowered this criterion to $2.0 \mathrm{mg} / \mathrm{dL}$ because from our clinical experience, many clinically septic patients presented with lactic acid levels in this range.

The EMR system generated sepsis alerts in real time as soon as combinations of findings fulfilled defined criteria. The system assessed data elements asynchronously; combinations of values from differing time points could be combined to activate an alert. Each fulfillment of additional sepsis criteria would result in the repeat activation of a sepsis alert. Vital signs were based upon nursing assessments entered into the EMR system. The hospital laboratory computer system (HealthQuest Data Systems, Highland, California) provided all laboratory test results.

While children have different ranges for SIRS criteria, $<1 \%$ of ED patients were $<18$ years old. (Goldstein et al. 2005) Therefore, we did not modify the sepsis alert rules by patient age. 
Automated sepsis screening occurred for all ED patients. The data for this study originated from a 3-month pilot testing period January 3, 2012 to March 31, 2012. During this period automated alerts were generated and evaluated post hoc, but were not communicated to clinicians.

Determination of the true diagnostic accuracy of the sepsis alert system would require manual review of ED records for all patients that did not activate the sepsis detection system. However, this would require manually reviewing over 18,000 ED medical records, which was not logistically feasible. In the effort to provide some comparison between sepsis alert and non-alert patients, we randomly selected 300 patients treated in the ED during the study period but who did not activate the EMR sepsis detection system. We chose this number based upon the availability of resources for manual medical record review.

\section{Outcomes - Confirmation of Sepsis}

To confirm the presence or absence of sepsis in each ED patient, two investigators manually reviewed the ED medical records for all sepsis alert activations and the randomly selected nonalert cases. We defined sepsis as the presence of 1) a serious infection related to the ED presentation, 2) $\geq 2$ SIRS criteria, and 3) systolic blood pressure $\leq 90 \mathrm{~mm} \mathrm{Hg}$ or lactic acid level $\geq 2.0 \mathrm{mg} / \mathrm{dL}$. We used previously published criteria to classify an infection as a "serious infection."(Angus et al. 2001; Wang et al. 2007) The presence of a serious infection was based upon ED clinician documentation, including the clinical narrative as well as ED diagnoses. We did not use laboratory or radiologic test results to confirm the presence of an infection. Because of our focus on the ED presentation, course of care, and clinical impression, we did not use medical records from later points of hospitalization nor discharge diagnoses to determine the 
106 presence of an infection. As part of the chart review process, the reviewers also confirmed the

107 fulfillment of SIRS criteria by each automated alert. Therefore, reviewers were not blinded to 108 presence or absence of a sepsis alert activation.

109 The reviewers resolved all discrepancies by consensus. In a test series of 30 records, inter-rater 110 agreement for the presence of sepsis was high (kappa=0.78).

111 Data Analysis

112 We determined the diagnostic accuracy of the automated EMR sepsis detection system by 113 calculating positive predictive value (PPV) of the sepsis alerts. We estimated the negative 114 predictive value based upon the sample of non-sepsis alert patients. Because of the sampled 115 nature of the non-alerts, it was not possible to calculate the sensitivity, specificity and area under 116 the ROC curve. We identified the infection category for true-positive sepsis alerts. We determined 117 the chief reason for ED visit for false-positive sepsis alerts and true-negative non-sepsis alerts.

118 We also determined the disposition of each patient (admitted to hospital, died in ED or discharged 119 home from ED). We conducted all analyses using Stata v.12.2 (Stata, Inc., College Station, 120 Texas). 


\section{RESULTS}

122 During the three-month study period, there were activations of the EMR sepsis alert system for 123795 ED patients. The mean age of sepsis alert patients was $55 \pm 20$ years, and half were male $124(51 \%)$. There was a total of 1,224 alerts across the 795 patients. The median number of alerts was 1252 per patient (IQR 1-2). The maximum number of alerts for an individual patient was 6.

Of the 795 EMR sepsis alerts, manual record review confirmed the presence of sepsis in 355 cases. (Table 1) The positive predictive value of the sepsis alert system was $44.7 \%$ (95\% CI: 41.2- 48.2\%). Among true-positive sepsis alerts, the most common infections were those of the 129 respiratory and urinary tract. (Table 2) Among the false-positive sepsis alerts, trauma, non-infectious 130 gastrointestinal disorders and cardiovascular disorders were the most common conditions. (Table 3)

Of the 300 randomly selected non-sepsis alert patients, none exhibited sepsis on manual chart review (estimated negative predictive value 100.0\%; 95\% CI: 98.8-100.0\%). The true negative non-sepsis alerts included a range of patients with infections that did not fulfill SIRS criteria. (Table 4)

134 Among true-positive sepsis alert patients, over 90\% were admitted to the hospital or died in the 135 ED. (Table 5) False-positive sepsis alert patients also exhibited high rates of hospital admission 136 or ED death. Few non-sepsis alert patients were admitted to the hospital. 
Over the three-month study period, this novel ED sepsis alert system was activated 795 times, identifying nearly 300 confirmed sepsis cases. Our results suggest that an EMR-based sepsis alert system could be used to identify sepsis patients in the ED.

The number of false positive sepsis alerts in this series is not clinically excessive. The clinical identification of sepsis is extremely difficult, requiring assimilation of clinical, physiologic and laboratory data.(Jaimes et al. 2003) Anecdotal data suggest that clinicians often under-detect sepsis cases. Jones et al. found that in a survey of emergency medicine physicians at 30 academic tertiary care hospitals, only $7 \%$ reported implementing early goal-directed therapy for sepsis, and the primary reason for this low rate was due to the poor identification of sepsis. (Jones \& Kline 2005) Other studies have shown that automated detection of medical conditions like abdominal aortic aneurysm and central line-associated blood stream infections is more effective than by manual surveillance alone.(Padberg et al. 2009; Woeltje et al. 2011) Our observations indicate that almost one in two sepsis alerts will be associated with a true sepsis case. Thus, the system offers aid in the identification of sepsis cases but with only a modest number of false positives. While we could not formally calculate the sensitivity of the system, the random sample of nonalert patients resulted in no sepsis cases, suggesting that the prevalence of false-negatives (undetected sepsis) may be low.

The number of false-positive sepsis alerts is not surprising given that many non-infectious medical conditions can present with vital signs and laboratory abnormalities that fulfill SIRS crtieria. For example, patients with cardiovascular, respiratory and even toxicologic conditions may present with tachycardia, tachypnea, or leukocytosis. Patients with trauma may exhibit 
tachypnea and tachycardia secondary to pain. Elevated lactic acid may be present in a range of conditions due to tissue hypoxia and subsequent anaerobic metabolism.(Bakker et al. 1996)

However, a notable observation was the high proportion of hospital admissions $(>80 \%)$ among the false-positive sepsis alerts, which was similar to that of the true-positive alerts. This finding suggests that the majority of cases activating a sepsis alert were high acuity patients. Therefore, the sepsis detection system may in fact have broader applicability as a general indicator of ED patient acuity. Further study is needed to characterize this latter population and to better delineate how the information might be integrated into ED clinical practice.

Prior studies have evaluated the use of EMR clinical decision tools to identify sepsis. Nelson, et al. evaluated the use of an automated surveillance algorithm at the University of Michigan Hospital, classifying sepsis as individuals with $\geq 2$ SIRS criteria plus systolic blood pressure of $\leq 90$. The system demonstrated a sensitivity of $64 \%$, PPV of $54 \%$, and NPV of $99 \%$ for detecting severe sepsis with signs of organ dysfunction.(Nelson et al. 2011) Our study enhanced the Nelson, et al. criteria by adding elevated lactate $(\geq 2.0 \mathrm{mg} / \mathrm{dL})$ as an additional inclusion criterion. As expected, this strategy increased the number of detected sepsis cases but at the cost of additional false positives (decreased PPV). Also, the Nelson study was based upon only 1 week of ED visits. Our study included a broader range of ED patients from a 3-month time frame.

Variations of the studied sepsis detection system have been developed for the inpatient setting, incorporating additional laboratory values such as coagulation and hepatic function panels. For this effort, we resisted using these extra values because of the likely increase in the number of false positive sepsis alerts. Also, the combination of SIRS criteria with hypotension or lactate is a widely recognized and accepted paradigm in Emergency Medicine; we believed that the selection of additional variables would introduce confusion in clinical application. We believe that the most 
182 important strategy for improving the system's accuracy is to incorporate automated methods for

183 identifying infections. Currently, the presence of infection is dependent upon clinician

184 interpretation and documentation. Biomarkers such as procalcitonin may potentially complement

185 sepsis detection efforts; a recent study demonstrated that procalcitonin had an excellent NPV

186 (96\%) and good sensitivity (75\%) and specificity (71\%) for identifying bacteremia and

187 pneumonia.(Albrich \& Mueller 2011; Torres et al. 2012) Future studies must evaluate these and

188 other strategies. 
190 Due to logistical limitations, we were not able to examine all non-alert ED patients; as discussed 191 previously, this would have required manual review of 18,000 records. However, our comparison 192 with randomly selected controls offered important insights, including the low rates of false 193 negatives. Examination of a larger series would likely have affirmed a higher NPV. The EMR 194 system depended on manual input of vital signs by ED personnel. Delayed or erroneous entries 195 may have altered alert activation patterns. Because of the low number of pediatric patients, we 196 did not study modified sepsis criteria for children.(Goldstein et al. 2005) We focused on sepsis 197 presenting to the ED setting - not sepsis developing later in the hospital course.

198 This study also examined the accuracy of automated sepsis detection but not its clinical 199 implementation. ED personnel reaction to sepsis alert data was not an a priori objective of this 200 study but is clearly an extremely important factor that merits additional study. An important

201 future study is to determine how activated prompts from the decision support system to the 202 clinician may increase the number of recognized sepsis cases in clinical practice. 


\section{CONCLUSION}

204 This ED EMR clinical support system identified patients presenting to the ED with sepsis.

205 Automated EMR sepsis detection may provide a viable strategy for ED sepsis identification. 


\section{REFERENCES}

Albrich WC, and Mueller B. 2011. Predicting bacteremia by procalcitonin levels in patients evaluated for sepsis in the emergency department. Expert Rev Anti Infect Ther 9:653-656.

Angus DC, Linde-Zwirble WT, Lidicker J, Clermont G, Carcillo J, and Pinsky MR. 2001. Epidemiology of severe sepsis in the United States: analysis of incidence, outcome, and associated costs of care. Crit Care Med 29:1303-1310.

Annane D, Bellissant E, and Cavaillon JM. 2005. Septic shock. Lancet 365:63-78.

Bakker J, Gris P, Coffernils M, Kahn RJ, and Vincent JL. 1996. Serial blood lactate levels can predict the development of multiple organ failure following septic shock. American Journal of Surgery 171:221-226.

Goldstein B, Giroir B, and Randolph A. 2005. International pediatric sepsis consensus conference: definitions for sepsis and organ dysfunction in pediatrics. Pediatr Crit Care Med 6:2-8.

Jaimes F, Garces J, Cuervo J, Ramirez F, Ramirez J, Vargas A, Quintero C, Ochoa J, Tandioy F, Zapata L et al. . 2003. The systemic inflammatory response syndrome (SIRS) to identify infected patients in the emergency room. Intensive Care Med 29:1368-1371.

Jones AE. 2006. Evidence-based therapies for sepsis care in the emergency department: striking a balance between feasibility and necessity. Acad Emerg Med 13:82-83.

Jones AE, and Kline JA. 2005. Use of goal-directed therapy for severe sepsis and septic shock in academic emergency departments. Crit Care Med 33:1888-1889.

Levy MM, Fink MP, Marshall JC, Abraham E, Angus D, Cook D, Cohen J, Opal SM, Vincent JL, Ramsay G et al. . 2003. 2001 SCCM/ESICM/ACCP/ATS/SIS International Sepsis Definitions Conference. Intensive Care Med 29:530-538.

Levy S, and Heyes B. 2012. Information systems that support effective clinical decision making. Nurs Manag (Harrow) 19:20-22.

Nelson JL, Smith BL, Jared JD, and Younger JG. 2011. Prospective trial of real-time electronic surveillance to expedite early care of severe sepsis. Ann Emerg Med 57:500-504.

Padberg FT, Jr., Hauck K, Mercer RG, Lal BK, and Pappas PJ. 2009. Screening for abdominal aortic aneurysm with electronic clinical reminders. American Journal of Surgery 198:670674.

Rivers E, Nguyen B, Havstad S, Ressler J, Muzzin A, Knoblich B, Peterson E, and Tomlanovich M. 2001. Early goal-directed therapy in the treatment of severe sepsis and septic shock. $N$ Engl J Med 345:1368-1377. 
239 Torres A, Ramirez P, Montull B, and Menendez R. 2012. Biomarkers and community-acquired

240

241

242

243

244

245

246 pneumonia: tailoring management with biological data. Semin Respir Crit Care Med 33:266-271.

Wang HE, Shapiro NI, Angus DC, and Yealy DM. 2007. National estimates of severe sepsis in United States emergency departments. Crit Care Med 35:1928-1936.

Woeltje KF, McMullen KM, Butler AM, Goris AJ, and Doherty JA. 2011. Electronic surveillance for healthcare-associated central line-associated bloodstream infections outside the intensive care unit. Infect Control Hosp Epidemiol 32:1086-1090. 


\section{Table 1 (on next page)}

Emergency Department (ED) automated sepsis alerts, January 1, 2012 - March 31, 2012.

Includes 795 ED visits with triggered sepsis alert. Table includes comparison with 300 randomly selected ED patients that did not trigger a sepsis alert. Positive predictive value of sepsis alert is $44.7 \%$ (95\% Cl: $41.2-48.2 \%)$. 


\section{TABLE 1}

Emergency Department (ED) automated sepsis alerts, January 1, 2012 - March 31, 2012.

Includes 795 ED visits with triggered sepsis alert. Table includes comparison with 300 randomly selected ED patients that did not trigger a sepsis alert. Positive predictive value of sepsis alert is $44.7 \%$ (95\% CI: 41.2-48.2\%).

\section{Confirmed Sepsis}

\begin{tabular}{cccc}
\hline Sepsis Alert & Sepsis & No Sepsis & Total \\
\hline Yes & 355 & 440 & 795 \\
No & 0 & 300 & 300 \\
\hline Total & 293 & 802 & 1095 \\
\hline
\end{tabular}




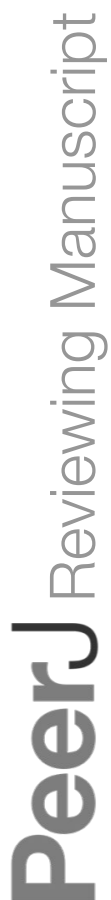

PeerJ reviewing PDF | (v2013:10:936:2:0:NEW 19 Mar 2014) 


\section{Table 2 (on next page)}

Infection types of Emergency Department visits with triggered sepsis alert and confirmed sepsis (true positive alert)

Total of $n=355$ true positive sepsis alerts. A patient may have had more than one infection. 


\section{TABLE 2}

Infection types of Emergency Department visits with triggered sepsis alert and confirmed sepsis (true positive alert). Total of $n=355$ true positive sepsis alerts. A patient may have had more than one infection.

\begin{tabular}{|c|c|}
\hline Infection Type & n (\%) \\
\hline $\begin{array}{l}\text { Pneumonia or Other } \\
\text { Respiratory }\end{array}$ & $107 \underline{135}(36.5 \underline{38.0})$ \\
\hline Urinary Tract & $104-\underline{116}(35.5 \underline{32.7})$ \\
\hline Gastrointestinal Bacteremia & $\underline{54(15.2)} 44(15.01)$ \\
\hline$\underline{\text { Bacteremia }}$ & $\underline{49(13.8)}$ \\
\hline Gastrointestinat & $43(14.7)$ \\
\hline Cellulitis & $25 \underline{\underline{33}}(8.59 .3)$ \\
\hline Abscess & $\underline{26(7.3)}$ \\
\hline Gynecologic & $5(1.47)$ \\
\hline Central Nervous System & $2 \underline{3}(0.7 \underline{9})$ \\
\hline Other Infection & $10-\underline{12}(3.4)$ \\
\hline
\end{tabular}




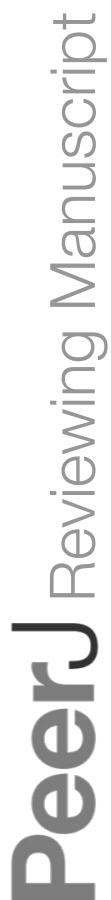

PeerJ reviewing PDF | (v2013:10:936:2:0:NEW 19 Mar 2014) 


\section{Table 3 (on next page)}

Medical conditions of Emergency Department visits with triggered sepsis alert but not confirmed sepsis (false positive alert).

Total of $n=440$ false positive sepsis alerts. A patient may have had more than one medical condition. 


\section{TABLE 3}

Medical conditions of Emergency Department visits with triggered sepsis alert but not confirmed sepsis (false positive alert). Total of $n=440$ false positive sepsis alerts. A patient may have had more than one medical condition.

\begin{tabular}{|c|c|}
\hline Medical Condition & $\mathbf{N}(\%)$ \\
\hline Gastrointestinal & $115(2 \underline{6.12} .9)$ \\
\hline Trauma & $\frac{112 \underline{113}}{(22.325 .7)}$ \\
\hline Cardiovascular & $88(17.5 \underline{20.0})$ \\
\hline Respiratory & $43(8.6 \underline{9.8})$ \\
\hline Overdose/Intoxication & $42(8.4 \underline{9.6})$ \\
\hline Central Nervous System & $39(\underline{8.97} .8)$ \\
\hline Renal & $34(\underline{7.76 .8)}$ \\
\hline Hematologic-Oncologic & $15(3 . \theta \underline{4})$ \\
\hline MiseeHaneotsother & $\begin{array}{l}120-119 \\
(27.13 .9)\end{array}$ \\
\hline
\end{tabular}




\section{Table 4 (on next page)}

Medical conditions of Emergency Department visits without triggered sepsis alert and without confirmed sepsis (true negative alerts).

Sample includes a total of $n=300$ patients not triggering a sepsis alert. A patient may have had more than one medical condition. 


\section{TABLE 4}

Medical conditions of Emergency Department visits without triggered sepsis alert and without confirmed sepsis (true negative alerts). Sample includes a total of $n=300$ patients not triggering a sepsis alert. A patient may have had more than one medical condition.

\begin{tabular}{|c|c|}
\hline Medical Condition & $\mathbf{N}(\%)$ \\
\hline$\underline{\text { Urinary Tract Infections }}$ & $\underline{27(9.0)}$ \\
\hline$\underline{\text { Respiratory Infections }}$ & $\underline{25(8.3)}$ \\
\hline$\underline{\text { Abscess }}$ & $\underline{8(2.7)}$ \\
\hline$\underline{\text { Cellulitis }}$ & $\underline{5(1.7)}$ \\
\hline$\underline{\text { Gastrointestinal Infections }}$ & $\underline{4(1.3)}$ \\
\hline$\underline{\text { Gynecologic Infections }}$ & $\underline{4(1.3)}$ \\
\hline$\underline{\text { CNS Infections }}$ & $\underline{0(0.0)}$ \\
\hline$\underline{\text { Bacteremia }}$ & $\underline{0(0.0)}$ \\
\hline$\underline{\text { Other Infections }}$ & $\underline{21(7.0)}$ \\
\hline Trauma & $\begin{array}{r}43-\underline{42} \\
(14 . \underline{3} \underline{0})\end{array}$ \\
\hline Non-Iinfection Gastrointestinal Conditions & $30(10)$ \\
\hline Urinary Traet Infeetions & $27(9.0)$ \\
\hline Respiratory Infeetions & $25(8.3)$ \\
\hline Other Infections & $20(6.7)$ \\
\hline Non-Infection CNS & $16(5.3)$ \\
\hline Soft Tissue Infeetions & $5(1.7)$ \\
\hline Drug Overdose & $11(3.7)$ \\
\hline
\end{tabular}


Cardiovascular Conditions

$8(2.7)$

Non-Infection Respiratory

Gastrointestinal Infeetions

Gynecologic Infections

Non-Iinfection Renal

Hematologic-Oncologic

Non-Infection Other
$3(1.0)$

$4(1.3)$

$4(1.3)$

$4(1.3)$

$1(0.3)$

$135-\underline{136}$

$(45.0 \underline{\underline{3}})$ 


\section{Table 5 (on next page)}

Emergency Department disposition of true-positive sepsis alert, false-positive sepsis alert, and non-sepsis alert patients. 


\section{TABLE 5}

Emergency Department disposition of true-positive sepsis alert, false-positive sepsis alert, and non-sepsis alert patients.

\begin{tabular}{lccc}
\hline & \multicolumn{3}{c}{ Type of Sepsis Alert } \\
$\begin{array}{c}\text { Emergency Department } \\
\text { Disposition }\end{array}$ & $\begin{array}{c}\text { True-Positive } \\
\text { Sepsis Alert } \\
\text { N (\%) }\end{array}$ & $\begin{array}{c}\text { False-Positive } \\
\text { Sepsis Alert N } \\
(\%)\end{array}$ & $\begin{array}{c}\text { No Sepsis } \\
\text { Alert N (\%) }\end{array}$ \\
\hline Admitted to Hospital & $323(91.0)$ & $365(83.0)$ & $17(5.7)$ \\
Died in ED & $1(0.3)$ & $1(0.2)$ & $0(0.0)$ \\
Discharged from ED & $31(8.7)$ & $74(16.8)$ & $283(94.3)$ \\
\hline
\end{tabular}

